\title{
Reaching your end-user with MIMAS
}

\author{
Abstract: \\ In the last five years the exponential \\ growth and use of electronic resources \\ has surpassed all former predictions. The \\ rise in use of e-journals, bibliographic \\ databases and research support facilities \\ has resulted in the researcher relying far \\ more heavily on web based resources than \\ ever before. One of the acknowledged \\ problems that now faces the researcher is \\ how to filter, access and apply the information, using the \\ wide variety of resource discovery tools now available to \\ them. At MIMAS (Manchester Information and Associated \\ Services, one of the three UK national data centres) we \\ attempt to provide a one-stop-shop for our users' needs, be \\ they undergraduate, postgraduate or post-doctorate. This \\ paper seeks to show how MIMAS services can be used at \\ every stage of the research development process, from the \\ inception of an idea, through its development, to the final \\ presentation of results. Facilities like ISI's Web of Science, \\ COPAC (which merges online catalogues of 21 of the \\ largest university research libraries in the UK and Ireland), \\ JSTOR (a retrospective digital archive of scholarly \\ journals) together with many of the other services hosted at \\ MIMAS now provide support for researchers at every level \\ of the creative process. \\ The world of scholarly writing has proven itself to be \\ a highly dynamic environment. Researchers are now \\ expected to be literate in an ever increasing number of \\ information seeking skills with the emergence of the \\ internet as a new medium for resource location. The ability \\ to search across huge databases, datasets and archives \\ is now possible, in a way that would never have been \\ conceivable to the first researchers. One thing that has \\ not changed is the basic structure of a written piece of \\ research. Some sections may be slightly larger than others \\ but in principle, there are six separate stages in the process \\ of creating research, which can be identified. Two authors \\ clearly identify these stages. \\ Thomas and Brubaker describe the structure of re- \\ search papers in the following way. 'The most popular \\ six chapter structure for the dissertation in use today \\ consists of (a) introduction, (b) review of the literature, \\ (c) methodology, (d) results/ findings, (e) analysis and \\ interpretation of findings, and (f) summary, conclusions, \\ applications and recommendations for \\ further study.' \\ Edminster and Moxley concur with \\ this description of research structure. \\ 'Although some modification of the six \\ chapter format occurs in dissertations \\ in the humanities, its influence is still \\ clearly visible in most graduate work \\ and the view that such work represents \\ original thought by an individual author who merits \\ recognition and reward for that originality continues to \\ prevail.' ${ }^{\prime 2}$ \\ One question we must ask ourselves is, with the basic \\ structure in place for the paper, why do some researchers \\ struggle in creating their research paper? Rosenfeldt and \\ Dowling refer to the tyranny of the blank page. ${ }^{3}$ Research \\ into the answer to this question can be found in literature \\ on the creation of training programmes, studies into user \\ searching skills, and many of the library and information \\ journals have examined various facets of this question. \\ I shall briefly look at some of the main causes for this \\ problem. \\ The most commonly recognised cause is computer anxiety. \\ Computer anxiety can occur in three distinct forms. These \\ are recognised by Torkzadeh and Angulo in their paper \\ in 1992. "a) Psychological anxiety which includes fear of \\ damaging the computer; b) sociological anxiety, which is \\ characterized by the need for social contact and the fear \\ of being replaced by a machine and c) operational anxiety, \\ which includes an inability to type and/or operate the \\ machine."4 \\ Another reason for difficulty could be the sea of \\ information now available to the researcher. The \\ presentation and manipulation of information is now \\ possible in such a huge variety of ways that it is no wonder \\ many scholars are intimidated at the onset. Many suffer \\ with feelings of information overload. Coupled with this \\ there are so many new initiatives and projects providing \\ new ways into the variety of data available to the scholar \\ that where to start can be the greatest challenge. Stewart \\ highlights the dilemma researchers now face, ' $\ldots$ in this \\ technological and techno phobic environment, novices are \\ faced with a variety of systems with different interfaces,
}


search capabilities, commands, and screen layouts among other options. ${ }^{5}$

The amount of information available is not the only significant challenge which the change in scholarly writing has faced. The range of skills users need to seek the information they require has continued to diversify. Researchers are no longer just required to be able to read and write but to be able to search across a variety of mediums from the book and journal to the Internet, the world wide web and the huge range of searching tools which are now available. With growing numbers of mature and foreign students the diversity of their capabilities to manage in this environment is another challenge that service providers must consider. The ranges of abilities from novice to expert all require different levels of help. Diane Nahl discusses this in her paper, 'Since the mid1980s, complex information retrieval systems have been available in academic libraries. As a result librarians have dealt with increasing numbers of novice end-users needing instruction in the use of database systems. At the turn of the century, the fast growing technological environment continues to introduce challenges for reference and instruction librarians who strive to help novices acquire effective search behavior.' 6

To allow this paper to have a focus I decided to select a topic that falls within all three major disciplines. The topic is the development of psychiatry. This topic can be taken from a scientific, a social scientific or a humanities angle depending on the researcher's emphasis. The reason why I have selected a cross disciplinary topic is due to the changing nature of research today. Research no longer falls neatly within the old ideas of specific discipline but now calls upon a wide range of cross-disciplinary information. Many of the tools I will discuss have acknowledged this change and allow cross-disciplinary searching. For our topic area the science researcher could view it as the development of psychiatry from a medical point of view. In the same way a social scientist may view this topic as the development of psychiatry as a social awareness topic. The humanities student could also look at this topic on the grounds of a historical project. Lois Buttlar in her discussion of the information sources for doctoral research discusses the importance of the understanding of cross-discipline searching. She refers to Chubin's work in this area. '... Chubin (1976) discusses Bradford notions of "core and scatter" noting that while a discipline is centred around an intellectual core, knowledge about communication outside the core (or scatter) indicates how the disciplines overlap. It is important for information professionals to understand the dynamics of knowledge overlap in research, especially since the hypertext technology has facilitated cross-disciplinary exchanges. ${ }^{7}$

The aim of this paper is to highlight how the crossdisciplinary services available at MIMAS can be used in the creation of a research paper. I shall illustrate how the researcher can draw on resources, which fall into a variety of disciplines, can be used throughout the research process and also make suggestions for their hypothetical use in a mock paper.

In the following paper I shall illustrate how the resources of one data centre can be used to enhance, support and develop a researcher's paper. My aim is not to discuss how one data centre or one archive of information is better than another but simply to show how a wide variety of services covering cross-disciplinary data can be used in the creation of a single research paper. In this paper I will be looking at the MIMAS data centre. In the UK there are three data centres: MIMAS (Manchester Information and associated services) based at Manchester University, EDINA (Edinburgh Data and Information Access) and BIDS (Bath Information Data Services)

MIMAS is a JISC supported national data centre run by Manchester Computing, at the University of Manchester, to serve the UK academic community. The range of services, hosted at MIMAS, provides flexible online access to socioeconomic, spatial and scientific data, and to bibliographic and electronic journal data services. The data service had previously been called MIDAS (Manchester Information Data Sets) but due to copyright law the name was changed to MIMAS in July of 1999.

I will briefly run through each of these services before looking at how they may be applied at the different stages of creating a piece of research. The services hosted at MIMAS can be broken down into six separate sections. The bibliographic reference services include four services, Archives Hub, COPAC, ISI Web of Science and ZETOC. The Electronic journal services include JSTOR and NESLI. Scientific Services include Crossfire, CSD and Mossbaur but I will only be looking at CasWeb. The socio-economic data include census data that is accessed through CasWeb, surveys, NS Databanks and international data. Spatial Data is made available in Map data and satellite data.

The Archives Hub service revolutionizes access to the archive collections held in UK universities and colleges by making descriptions of them available on the Internet, many for the first time. There are currently over 40 institutions contributing to the collection. Records listed on the service cover a wide range of sources, including papers of statesmen, scholars, soldiers, scientists and storytellers. The current descriptions are just "top-level", giving an overview of each collection, with a brief biography of the creator of the documents, a note on the contents of the archive and idea of the quantity of material concerned. Where possible this top-level description is linked to a full catalogue and each archive description is indexed to aid researchers in locating relevant material. 
COPAC provides free access to the merged catalogues of major UK and Irish university research libraries. Within the database there are over 8 million records from the 22 contributing libraries, with more being loaded. Records found in COPAC represent a variety of materials across most subject areas. Some include links to full-text documents made available by other services. The materials range in date from c. $1100 \mathrm{AD}$ to the most recent documents with 300 languages being represented. Further to the bibliographic details of the records, COPAC also has realtime local availability information that has been provided for some libraries. Records can be downloaded via email and imported into reference management software.

The Web of Science is the web interface providing access to ISI's three central databases. These are: Science Citation Index, Social Sciences Citation Index, Arts \& Humanities Citation Index, and the interface was designed by the Institute for Scientific Information (ISI). The data in these databases covers articles dating back to 1981. ISI Proceedings, the sister service of Web of Science, has two databases, the Science and Technical edition (STP) and the Social Science and Humanities edition (SSHP). Coverage in ISI Web of Science and ISI Proceedings continues to grow. Currently there are more than 8500 journals and records of nearly 2 million papers in proceedings have been indexed by ISI, and over 20,000 new items are added each week. In addition to these two central services, the Web of Science service also provides access to additional ISI products. These include Current Contents Connect, Journal Citation Reports, Derwent Innovations Index, ISI Chemserv and Biosis Previews.

ZETOC provides Z39.50-compliant access to the British Library's Electronic Table of Contents (ETOC). The service was launched in September 2000. The database contains over 19 million article titles from over 20,000 of the most important research journals and 16,000 conference proceedings, covering every imaginable subject in science, technology, medicine, engineering, business, law, finance and the humanities. Records start from 1993 and the database is updated daily with approximately 10,000 new records. The service also allows users to send themselves alerts when new issues of journals arrive. This is known as Zetoc alert. MacIntyre and Apps describe this service, 'To supplement the basic search and retrieval functionality of the service, a current awareness alerting service based on the table of contents data was also developed. The aim of was to 'fill the gap' left by the demise of the 'Autojournals' service offered by BIDS, another of the UK's national academic datacentres, until July 2000.",

JSTOR was established as an independent not-for-profit organization in August 1995; it is a retrospective collection of electronic full text articles. The MIMAS based JSTOR service is a mirror service of the American based service. JSTOR combines the advantages of page images with searchable full-text, and stores the data in both forms. JSTOR has a full-text electronic archive collection of noncurrent issues of over 100 core scholarly journals covering a wide range of subjects. Coverage within the collection starts with the very first issues (many dating from 1800s). JSTOR continues to expand and in the last two years three new archive collections have been added to the service. The new collections are called Ecology \& Botany Collection, Arts \& Sciences II Collection and the Business Collection. This year we will see the launch of a new Language \& Literature collection.

NESLI delivers a national electronic journal service to the UK higher education and research community. The managing agent undertakes negotiations with journal publishers on behalf of UK Higher Education Institutions for electronic journals at preferential terms. Once a publisher has created a provisional offer it is then sent to the Journals Working Group or JWG before the final offer is sent out. Access to the subscribed journals is done in several ways. Institutions have the option, depending on the deal, to access the information either directly from the publisher's site or through the NESLI/SwetsnetNavigator WWW gateway. This gateway provides a single point of access to the full text of electronic journals. The range of journals available through this access point is dependent on the publisher's agreement to have access through this point. Site licenses are available for individual e-journals or packages of e-journals, depending on the current publisher's deal. For the period of 2002 there are currently 21 publisher deals in progress. Material covered by these deals is cross-disciplinary in nature.

CrossFire is a comprehensive chemical information management system comprising the CrossFire Beilstein and CrossFire Gmelin databases and the Beilstein Commander and CrossFire Web software which are used to search and access the databases. CrossFire Beilstein contains over 8 million organic compounds, with over 8 million reactions and over 4 million citations. CrossFire Gmelin contains over 1.5 million inorganic and organometallic compounds, 1.3 million reactions and 1 million citations.

CasWeb was originally developed by James Harris at the ESRC/JISC and funded by the Census Dissemination Unit (CDU), which forms part of the MIMAS service. CasWeb is just one piece of software found in the CDU but is most appropriate to this paper. I will not be discussing the CDU as this topic will be covered by my colleague, Jackie Carter at a later stage of this conference. CasWeb is a web interface to statistics and related information from the 1991 United Kingdom Census of Population. These large and complex datasets comprise aggregate counts of persons and households for various geographical units. The Census SAS/LBS datasets contain over 10,000 items of information, which are arranged for convenience into tables. The CasWeb system has preserved this arrangement 
of the data.

The Landmap project uses data from two different satellites, Landsat and SPOT. The Landsat, an American satellite, consists of 32 scenes covering the UK. The Landsat Data Maps allow users to view maps at 30m resolution. It is used in the study of marine environment, water resources, land use, ecological and engineering applications, agriculture and forestry monitoring. The SPOT, a French satellite, is composed of 152 scenes and covers the UK and Republic of Ireland. SPOT allows users to view Data Maps down to $10 \mathrm{~m}$ resolution. It is used in much the same way as Landsat but it is mainly concentrating on studies of land use mapping and digital terrain modeling.

As I have illustrated the MIMAS services cover a wide range of disciplines and can be applied to research in a variety of ways. Using the topic of the development of psychiatry I shall now show how these tools can be employed through the six stage process of creating a research paper.

\section{Introduction}

Rosenfeldt and Dowling describe this stage in the following way, "... Once you begin, you must face the tyranny of the blank page - a major cause of procrastination. The first step is to get something down. Begin with a plan." Most of the work done before hand is centred upon reaching the core of the research project. "Areas focused on here include drawing up a short list of topics, selecting a topic for investigation, formulating a general question and focusing a research question. The importance of spending as much time as necessary to get the question right is highlighted here as this often causes new researchers considerable difficulty." 10 It is the pre-cursor for the creation of your original idea, the core from where the research will start and finish. At this point the user reads a huge amount of literature to create this original idea. The introduction is the point where the net of research is the widest pulling in resources from cross-disciplinary sources. Portals like those provided at ensure that the articles, journals and other resources found at MIMAS are of highest quality and suitable for inclusion in a piece of scholarly writing.

MIMAS resources like COPAC are particularly useful at this stage. COPAC as a union catalogue of 22 libraries provides bibliographic details encompassing an enormous range of material ranging from maps to pictures to written texts. This allows the users to discover resources from a huge database of resources. The interface was designed to be as simple as possible. It is possible for users to search under author, keyword etc. In some cases articles in COPAC link straight through to the full text of articles. Alternatively, users of COPAC can view their local holding details of libraries and can arrange for interlibrary loans. A search on psychiatry produces a large set of results, totalling 12,485 , amongst these results we can see material including books, journal articles, medical textbooks, handbooks, illustrated texts and even lecture notes.

Another resource, which can be applied at this stage of the research process, is the Archives Hub. As I mentioned earlier the Archives Hub serves as a gateway for archives in the university sector. Archives of relevant material can be located using this resource. Information at the archive level includes details on the collection itself, but also details on the repository. Details include important telephone numbers, opening hours, etc. In this way the researcher can identify archive collections which may be useful to their research topic.

Web of Science is another useful tool that the researcher can utilize at this stage of the research process. For novice users this has proven to be a useful tool to help them through their research. Two functions of searching exist within the service. Full Search that allows users to run advanced searches using Boolean operators and more complex search queries. In Full Search it is possible to view 500 records at a time. The second search available is Easy Search; this allows users to search under Person, Place and Topic. When the researcher searches the database for Place, he can create a search to retrieve the most recent articles published by researchers working in a particular institution (college, university, company, etc.) or geographical area (country, city postal code, etc.). A search on Person, will produce results on the person named as an author or a cited author or on an individual you want information on. Topic searches allow the user to find articles that discuss the key topics in their research. This is a good starting place when the researcher is simply trying to come to grips with the research question. Results from a topic search are displayed sorted by relevance. This means that the system will list first those articles that contain the most frequent occurrences of the words and/or phrases entered by the user to describe the topic. Alternatively results can be displayed in reverse chronological order. This lists the most recent articles first based on the date on which the journal was processed at ISI.

ZETOC is another MIMAS tool, which can be applied at this phase of the paper. Zetoc can be used to search the tables of contents of journals hosted at the British Library. Zetoc can also be used to set up an alerting service that will notify the researcher when relevant journals he has recognized as useful to his research have been published. In using zetoc, researchers can ensure that the future work they do in their paper will be as up to date with theories in the field as possible prior to submission and or publication.

\section{Literature Review}

At this stage the focus of the research has been completed. The researcher now reads and gathers literature from the chief influences for the topic area. It is important that the 
material included in this section is accurate and of the highest possible quality and relevance to the research topic, and the research question. The researcher must use this section of the paper to demonstrate the relationship between the proposed research and what has already been done in the area.

Web of Science can be used in a slightly different way at this point. By using the Full Search as opposed to the Easy search the researcher can track authors' works and also the authors that have cited the author's work. We can also widen the number of articles by looking at related records. Related records allow you to view articles that have shared one or more of the articles used in the original article's reference list.

Web of Science also allows users to run cited reference searches. This resource allows users to track a particular work by an author through its citations. In this way the Web of Science can be used as a tree so users can move from the start of a particular author and track backwards through the inspiration of the paper in question. They can move from branch to branch from the root of a particular topic. In the same way the user can then look beyond the original paper, and see how the paper has influenced later authors on the topic in question. Users can use this unique method of moving along the branches of knowledge built up within the web of science.

Two services within the web of science allow users to move beyond the bibliographic records to the full text of an article. The first service is the Holdings Service. This service allows users to click on a button in the top right of the screen and link through to their host institution's OPAC and see if the article in question is hosted at their own library. In this way they have access to the hard copy of the text. The second service is called the Linkage Service. ISI makes it easy to link between the Web of Science and electronic full text. ISI's goal is to provide users with bi-directional links for navigation back and forth between records in the Web of Science and corresponding full-text documents and references. ISI Links provides reliable access to full text. A link button will only appear when there is an active link to a document. This "no dead links" policy insures that Web of Science users will only get links that resolve to publishers' electronic full-text documents. ISI actively seeks full-text linking partnerships with primary journal publishers and content hosts for all available electronic journals covered by the Web of Science. Currently, over 5.4 million record links are available between the Web of Science and electronic journals and databases. Currently ISI has links set up with 14 core publishers.

As I mentioned earlier in the paper, Web of Science has other allied tools from ISI, one of which is the Journal Citation Reports or JCR. This tool allows users to see which journals have been cited the most. This will help them determine which journals are the most pertinent and useful in the search for valued peer reviewed articles to support their work. This tool can be used in conjunction with zetoc during the creation of the journal alerting list.

COPAC can again be used at this stage of the research process. Now that the main authors on the topic have been identified, COPAC using the union catalogue can be used to track down less common articles and references. Using the author/ title search allows the user to be far more precise than on earlier searches. It is at this point that the holdings function in COPAC becomes most useful. At the base of a full record, COPAC has holdings material that will allow researchers to either ask for an ILL from a specific repository or travel to its location. In this way more obscure texts can be identified and located.

JSTOR can be called upon at this stage to look for articles that have retrospective influence on the research topic. Searches can be made as easy or as complex as the user wishes by building up searches on the search form. Users have the option of selecting to search journal topic groups in clusters or to expand the list and search on individual journal titles. This later option tends to produce more accurate search results. Once the list of results is compiled the researcher can click to view a pdf of the full text of the article. JSTOR uses high-resolution images to store, display and print faithful replications of the pages that make up the complete published record of the journals in its archive. Users can view the article in an electronic copy of its original format. The ability to view the full text has proven itself to be very popular as researchers increasingly look for faster access to the full text of articles.

NESLI is another route for the researcher to reach the electronic journal. NESLI uses the Swetsnet Navigator interface. Users can log on to the service and search through the full text electronic journals of the NESLI publisher offers which their institution has subscribed to. Users can search on journal title, publisher or they can run a general or an advanced search. The journal title and publisher searches allow the researcher to type in one word from the journal title or publisher name and it will search for relevant records. The general search allows users to enter a date range between 1996 to the current year. They can then enter search terms, which will look for articles on article title, author, and abstract or on keywords. The advanced search allows users to select a date range again but they can create a more complex search using Boolean operators. Once a list of results is created the researcher is then able to click through to the abstract or to the full text of the article which they can view in a number of formats. Most articles are viewable as a pdf. The article will include any images that authors have included in their original article. The researcher can then print these articles to read later. 


\section{Methodology}

The methodology section of the paper is used to give a detailed description of how the research question or questions will be answered through the paper. This section will identify the steps the research will follow in order to answer the original research question. This stage is only possible through the work completed in the prior two stages. This section also normally discusses the application of either qualitative or quantitative research methods. Qualitative research examines topics that cannot be examined through statistical analysis. This research area has been described as humanist, realist, subjectivist and observational. The researcher attempts to qualify reasons for certain behaviour and to explain their occurrence in a scientific way. Quantitative research relies on empirical data. This is normally gathered through a structured questionnaire and basic statistical analysis.

Searching for qualitative and quantitative articles to support whichever method the researcher plans to utilise can be done using several of the bibliographic tools that were used in the introductory section. By simply using these terms in Web of Science the researcher can view articles on both areas of methodology and look at different applications of these theories within varied disciplines. COPAC can again be used to track down articles not cited in the Web of Science. JSTOR can be used to look at developments since the origins of the research methodology question in the journal archives.

\section{Results/Findings}

The results and findings section of the paper is the point where the researcher must provide the proof of the research undertaken. Sources for his results and findings can come from an array of sources.

CasWeb is the most obvious service to be used at this stage of the research process. CasWeb allows an intuitive access point to the Census of 1991 using a familiar Windows-based system. The researcher can click through the application first searching on specific tables of data, which have been retained for ease of use mirroring the original census format. This is a huge change from the earlier SASPAC (small area statistics package) system that required users to have knowledge of the structure and content of the census data. In this way it acts as an extraction process. While the user is able to extract specific information from the census they would then have to move the data from the remote machine before they could make use of it. CasWeb in direct contrast works as an acquisition process. It enables the user to extract the information but acquire the information directly onto their local machine. In this way the data becomes far more easily accessible.

To access the data you follow a simple 3-stage process.

1. In Step 1 you define the study area you are interested in and select the geographical units for which you wish to extract data.

2. Step 2 is where you define what data you want to extract from the census database. The Census SAS/LBS datasets contain over 10,000 items of information

\section{Step 3 is the data extraction / download stage}

The result is the user has a route to a diverse array of data, which can be applied in many ways. For the purpose of our research topic use could be made of the data by looking at the number of psychiatric institutions that exist within a specific area and compare that data with figures found in past censuses for the same area. CasWeb also allows users to map the data they gather using ArcView or MapInfo. In this way users can produce a visual representation of psychiatric institutions at ward level, county level or countrywide level allowing for an alternative view of the data away from the census tables. This can help the researcher to interpret these figures in a different way.

An additional Web of Science product called Derwent Innovations Index can also be utilised at this stage of the paper. Derwent Innovations Index provides a unique access point into patent material over the last twenty years. Users can search through patents based on patent topic area and discover innovations within their desired subject area. For the purpose of this paper typing in a topic word like psychiatry produces over 100 hits. Within this material patents can be found on electrical equipment used for monitoring patients' responses to innovations in drugs for psychiatric patients. One of the results looks at the use of different coloured lenses within glasses to affect the mood of emotionally disturbed patients over long periods of time.

JSTOR could also be used at this point of the paper. Journal articles in JSTOR can be used to illustrate changes in technology and their application. Since the archive carries papers from their first edition the development of theories, instruments and the success of certain drugs can be traced within journal articles. These articles can be used to support or refute current beliefs in the psychiatric field. The archive also holds images which were included in the original paper. These pictures can be used to illustrate changes in technology and their application for treatment, through the centuries, of psychiatric patients.

Crossfire is a chemists' tool but can also be made useful for this paper. The researcher can use Crossfire to view the chemical structure of drugs that were prescribed to patients. The application provides highly detailed information on how to purify chemicals, their basic physical attributes and their reactions with other elements. If a paper concentrated on the development of chemical treatment of psychiatric patients this could prove to be a very useful tool. 
Landmap can be used at this stage to illustrate the layout of a specific geographical area. The satellite images allow a unique view of the land and geographical features. If we use this in conjunction with the data from CasWeb viewed in ArcView it can allow the researcher to provide another view of an area of interest. Hypothetically, if the research topic discussed a specific institution which was originally built at an earlier point in history, details recorded at that point, for example, A map of the institution and its surrounding areas could be compared with current satellite data. This could be used to illustrate the growth of an institution or to highlight changes to the surrounding areas influencing the growth of the institution.

\section{Analysis of Findings}

The analysis of findings is a section where the researcher compiles the knowledge, resources and understanding of the topic area, the discipline and his/her findings for the paper. It is not possible to suggest an appropriate MIMAS based resource tool, as the user will be applying the information gathered during the prior stages of the research process. To a greater or lesser extent this data will have been gathered from the resource tools I have already suggested, and it is in this way MIMAS resources have benefited the user at this section of the research topic.

\section{Summary/Conclusions}

The summary of the research paper is the final point where the researcher highlights how he has laid the case for the research question and provided answers for the query under discussion. It is at this point that the researcher culminates all of the work he has put into the paper and summarises how he has achieved the aims and goals he laid out in the introduction. This would not be possible without the influence of the tools he has applied throughout the project.

As you can see the MIMAS tools are cross disciplinary, and yet all can be used to greater or lesser extent in various stages of the research process. It is important that researchers feel comfortable in using these resources and that they are capable of making use of these information seeking tools. I have provided numerous hypothetical applications of these tools to illustrate the cross disciplinary nature of research today and provide some suggestions for how researchers can make use of our resources.

To conclude, MIMAS tools are multi-disciplinary. The researcher should not be intimidated by the variety available, but embrace them. As research continues to blur the old divisions of research between arts and social science and social science and science it is important that tools exist which encompass this move. The facilities at MIMAS have been designed to be as intuitive and user friendly as possible. Each service has an individual helpdesk that provides weekday support when users become confused or lost. Many of the applications utilise context sensitive help as a first port of call. All of the bibliographic software also has FAQs (Frequently Asked Questions) sections to try and answer some of the core common queries to help hesitant users from feeling intimidated in asking for help. Zetoc and COPAC share the same interface allowing for familiarity of the resource tool for users. Similarly the Web of Science and all of its additional products also use the same interface allowing for increased familiarity for the user on utilising the various products. CasWeb was designed specifically to be user-friendly. It heralded a move from the old SASPAK system to a familiar Windows interface with online help. MIMAS services have been created to help the end user find the information they seek using intuitive, clear and user-friendly tools. In this way MIMAS can be viewed as an excellent foundation with reliable resource tools to aid researchers to reach their goal of creating a high quality, insightful research project within their field of expertise.

Paper presented at the IASSIST Conference, June 2002, in Storrs, CT, USA.

* Jessica Eustace, University of Manchester, UK. E-mail: jess.eustace@man.ac.uk

\section{Bibliography:}

1. Barbara Brien, Beth Hillman and Victoria Topp. Effectiveness of hands-on instruction of electronic resources. Research Strategies 16 (1) 1998. pp41-51

2. Lois Buttlar. Information Sources in Library and Information Science Doctoral Research. Library and Information Science Research 21(2) 1999. pp227-245

3. Jude Edminster and Joe Moxley. Graduate education and the evolving genre of electronic theses and dissertations. Computer and Composition 116, 2002. pp116

4. Emily Fabiano, Casting the net: reaching out to doctoral students in education. Research Strategies 14 (1996), 159-68

5. Nancy Fjallbrant. Evaluation in a user education programme. Journal of Librarianship 9 (2) 1977. pp83-95

6. A.J Jerebek and Linda Meter. "Library Anxiety" and "computer anxiety": Measures, validity and research implications. Library and Information Science Research, 23, 2001. pp277-289

7. Ross MacIntyre and Ann Apps. Working with the British Library - the 'zetoc' experience. 2002. http. /epub.mimas.ac.uk/papers/macappslwow4.pdf

8. C. A. Mellon. Library Anxiety: A grounded theory and its development. College and Research Libraries 47, 1986. pp160-165 
9. Thomas R. Murray and Dale L. Brubaker. Theses and dissertations: A guide to planning, research and writing. Westport Beigin and Gurvey. 2000.

10. Diane Nahl. Creating user centred instructions for novice end-users. Reference Services Review 27(3) 1999. pp280-286

11. Jill Newby. Evolution of library research methods course for biology students. Research Strategies 17, 2000. pp57-62

12. Barbara Nelson. Evaluating electronic resources. Conference Report/ Library Collections, Acquisitions and Technical Service 24, 2000. Pp 403-441

13. D. Nunan, D. Research methods in language learning. Cambridge: Cambridge University Press. 1992. in Brian Paltridge. Thesis and dissertation writing: preparing ESL students for research. English for Specific Purposes 16 (1)

14. Steve O'Connor. Economic and intellectual value in existing and new paradigms of electronic scholarly communication. Library Hi Tech 18 (1) 2000. pp37-45

15. Anthony Onwuegbuzie. Writing a research proposal: The role of library anxiety, statistics anxiety, and composition anxiety. Library and Information Science Research. 19 (1) 1997. pp5-33

16. Ruth Pagell. Reaching for the bottle, not the glass; the enduser factor of electronic full text. Database 16 (5) 1993.

17. Sarah Porter. Into the future. Scholarly needs, current provision, and future directions. 2000. http: /ahds.ac.uk/public/uneeds/un5.htm1

18. F.L.Rosenfeldt, How to write a paper for publication. Heart, Lung and Circulation. 9, 2000. pp 82-87

19. L. Stewart. Helping students during online searches: an evaluation. Journal of Academic Librarianship. 18(6) 1993. pp 347-351

20. G Torkzadeh and I.E. Angulo The concept and correlates of computer anxiety. Behavior and Information Technology. 11, 1992. pp99-108

21. Bob Travica. Organizational aspects of the virtual library: a survey of academic libraries. Library and Information Science Research, 21 (2) 1999. pp173-203

22. Denise Troll. How and why the libraries are changing: what we know and what we need to know. Libraries and the Academy 2.1, 2002. pp99-123

\section{Footnotes}

${ }^{1}$ Thomas R. Murray and Dale L. Brubaker. Theses and dissertations: A guide to planning, research and writing. Westport Beigin and Gurvey.' 2000. p. 8

2 Jude Edminster and Joe Moxley. Graduate education and the evolving genre of electronic theses and dissertations. Computer and Composition 116, 2002. p.8

${ }^{3}$ F.L.Rosenfeldt, How to write a paper for publication. Heart, Lung and Circulation. 9, 2000. p. 83

${ }^{4}$ A.J Jerebek and Linda Meter. "Library Anxiety" and "computer anxiety": Measures, validity and research implications. Library and Information Science Research, 23 2001. p. 278

${ }^{5}$ L. Stewart. Helping students during online searches: an evaluation. Journal of Academic Librarianship. 18(6) p348

${ }^{6}$ Diane Nahl. Creating user centred instructions for novice end-users. Reference Services Review 27(3) 1999. p280

${ }^{7}$ Lois Buttlar. Information Sources in Library and Information Science Doctoral Research. Library and Information Science Research, 21(2) p.229

${ }^{8}$ Ross MacIntyre and Ann Apps. Working with the British Library - the 'zetoc' experience. 2002. http: /epub.mimas.ac.uk/papers/macappslwow4.pdf

${ }^{9}$ F.L.Rosenfeldt, How to write a paper for publication. Heart, Lung and Circulation. 9, 2000. p. 83

${ }^{10}$ D. Nunan, D. Research methods in language learning. Cambridge: Cambridge University Press. 1992. in Brian Paltridge. Thesis and dissertation writing: preparing ESL students for research. English for Specific Purposes 16 (1) p.63 


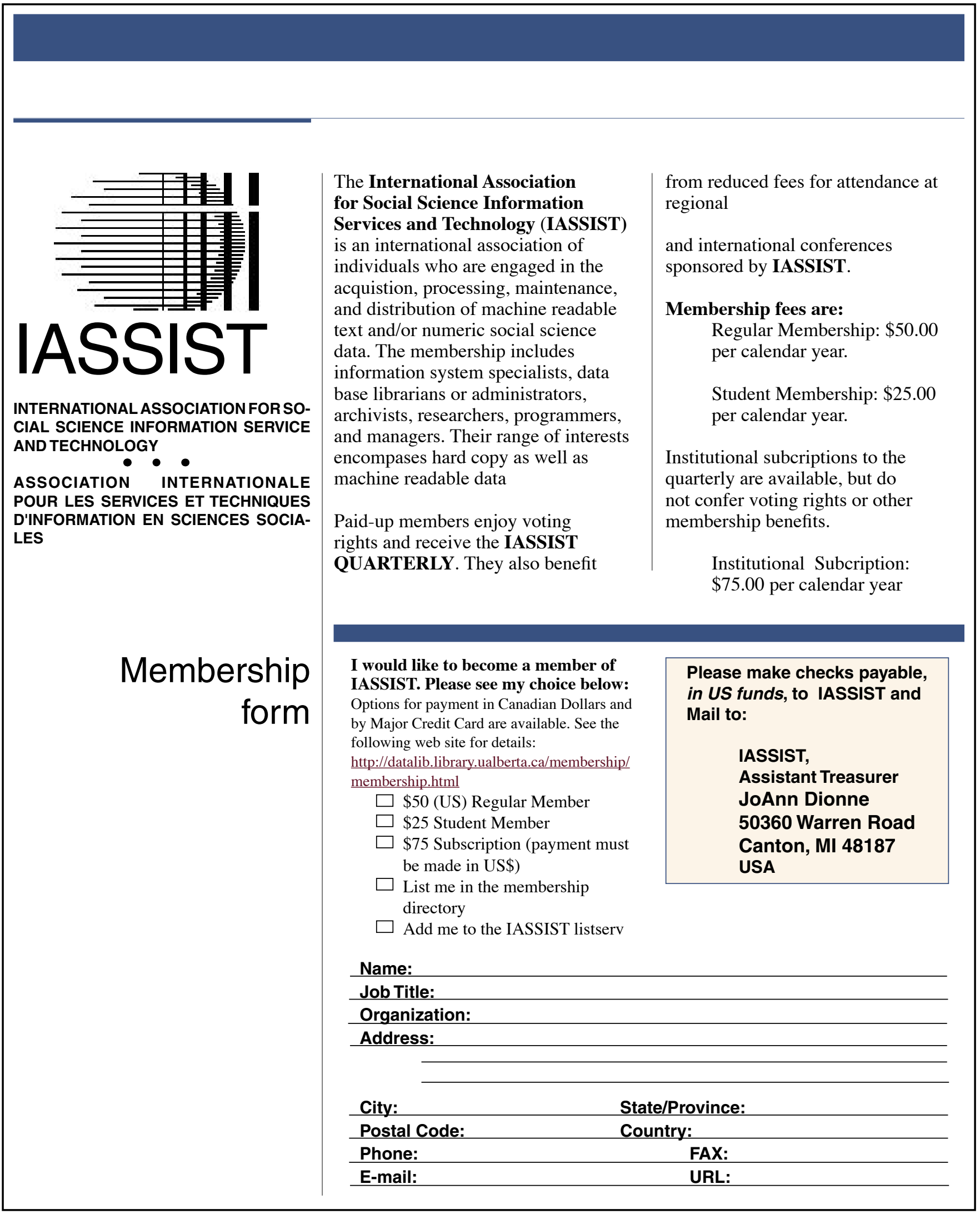

